\title{
Boundary value problems of fractional $q$-difference equations on the half-line
}

\author{
Kuikui Ma' ${ }^{1}$ Xinhui $\mathrm{Li}^{1}$ and Shurong Sun ${ }^{1 *}$
}

\section{"Correspondence:}

sshrong@163.com

'School of Mathematical Sciences,

University of Jinan, Jinan, P.R. China

\begin{abstract}
In this paper, we consider the boundary value problem of a class of nonlinear fractional $q$-difference equations involving the Riemann-Liouville fractional $q$-derivative on the half-line. By means of Schauder fixed point theorem and Leggett-Williams fixed point theorem, some results on existence and multiplicity of solutions are obtained.

MSC: 39A13; 34B18; 34A08

Keywords: Fractional $q$-difference equations; The half-line; Existence and multiplicity of solutions
\end{abstract}

\section{Introduction}

Quantum calculus, roughly speaking, is ordinary calculus without limits. There are several types of quantum calculus: $h$-calculus (also known as the calculus of finite differences), $q$ calculus, and Hahn's calculus. In this paper we are concerned with the $q$-calculus. The $q$-derivative and the $q$-integral were first defined by Jackson $[1,2]$ and had proven to have important applications in many subjects, like in hypergeometric series, complex analysis, particle physics, and quantum mechanics. For a general introduction to the $q$-calculus, we refer the reader to the book [3].

The origin of the fractional $q$-difference calculus can be traced back to the works in [4] by Al-salam and Agarwal. Perhaps due to the development of the fractional differential equations, an interest has been observed in studying boundary value problems of fractional $q$-difference equations, especially, about the existence of the solutions for the boundary value problems [5-10].

Boundary value problems on a half-line arise quite naturally in the study of radially symmetric solutions of nonlinear elliptic equations and models of gas pressure in a semiinfinite porous medium. Though much of the work on fractional calculus deals with finite domain, there is a considerable development on the topic involving unbounded domain [11-24].

In 2010, Zhao and Ge considered the following fractional boundary value problem [11]:

$$
\begin{aligned}
& D_{0+}^{a} u(t)+f(t, u(t))=0, \quad t \in(0, \infty), a \in(1,2), \\
& u(0)=0, \quad \lim _{t \rightarrow \infty} D_{0+}^{a-1} u(t)=\beta u(\xi),
\end{aligned}
$$

(c) The Author(s) 2019. This article is distributed under the terms of the Creative Commons Attribution 4.0 International License (http://creativecommons.org/licenses/by/4.0/), which permits unrestricted use, distribution, and reproduction in any medium, provided you give appropriate credit to the original author(s) and the source, provide a link to the Creative Commons license, and indicate if changes were made. 
where $0<\xi<\infty, D_{0+}^{a}$ is the standard Riemann-Liouville fractional derivative. By means of fixed point theorems, sufficient conditions are obtained that guarantee the existence of solutions to the above boundary value problem.

In 2011, Zhao et al. studied the fractional multi-point boundary value problem [14]

$$
\begin{aligned}
& D_{0+}^{a} u(t)+f(t, u(t))=0, \quad t \in(0, \infty), \\
& u(0)=0, \quad D_{q}^{a-1} u(+\infty)=\sum_{i=1}^{m} \alpha_{i} u\left(\xi_{i}\right),
\end{aligned}
$$

where $1<a \leq 2,0<\xi_{1}<\xi_{2}<\cdots<\xi_{m}<\infty, D_{0+}^{a}$ is the standard Riemann-Liouville fractional derivative. By Leggett-Williams fixed point theorem, sufficient conditions that guarantee the existence of three positive solutions are obtained.

To the best of our knowledge, there is no paper considering fractional $q$-difference equations on the half-line. Theories and applications seem to be just being initiated. In this paper we will fill in the gap to investigate the existence of solutions for the following boundary value problem of nonlinear fractional $q$-difference equations on the half-line:

$$
\left(D_{q}^{\alpha} u\right)(t)+f(t, u(t))=0, \quad 0 \leq t<\infty,
$$

subject to the boundary conditions

$$
u(0)=0, \quad D_{q}^{\alpha-1} u(+\infty)=\sum_{i=1}^{m} a_{i} u\left(\xi_{i}\right)
$$

where $0<q<1,1<\alpha<2,0 \leq \sum_{i=1}^{m} a_{i} \xi_{i}^{\alpha-1}<\Gamma_{q}(\alpha), f:([0, \infty), \mathbb{R}) \rightarrow[0, \infty)$ is a continuous function. Most results on the solution of boundary value problem of fractional $q$ difference equations that have been obtained concern the finite interval. In this paper, the range of variables is considered on the half-line. Since the Arzela-Ascoli theorem fails to work in the space $C_{\infty}$, we use a modified compactness criterion to prove $T$ is compact. We prove the existence and multiplicity results on positive solutions for boundary value problem (1.1)-(1.2) by utilizing Schauder fixed point theorem and Leggett-Williams fixed point theorem. Several existence results for solutions on the half-line are obtained. This work is motivated by papers $[11,14]$.

The paper is organized as follows. In Sect. 2, we introduce some definitions of $q$ fractional integral and differential operator together with some basic properties and lemmas to prove our main results. In Sect. 3, we investigate the existence of solutions for boundary value problem (1.1)-(1.2) by Schaefer fixed point theorem and LeggettWilliams fixed point theorem. In Sect. 4, we give an example to illustrate our main results.

\section{Preliminaries}

In the following section, we collect some definitions and lemmas about fractional $q$ integral and fractional $q$-derivative for the integrity of the theory, for which we refer to [25-27]. 
Definition 2.1 ([4]) Let $\alpha \geq 0$ and $f$ be a function defined on $[0, b]$. The fractional $q$ integral of the Riemann-Liouville type is defined by $\left(I_{q}^{0} f\right)(x)=f(x)$ and

$$
\left(I_{q}^{\alpha} f\right)(x)=\frac{1}{\Gamma_{q}(\alpha)} \int_{0}^{x}(x-q t)^{(\alpha-1)} f(t) d_{q} t, \quad \alpha>0, x \in[0, b] .
$$

Definition 2.2 ([26]) The fractional $q$-derivative of the Riemann-Liouville type of order $\alpha \geq 0$ is defined by $\left(D_{q}^{0} f\right)(x)=f(x)$ and

$$
\left(D_{q}^{\alpha} f\right)(x)=\left(D_{q}^{p} I_{q}^{p-\alpha} f\right)(x), \quad \alpha>0
$$

where $p$ is the smallest integer greater than or equal to $\alpha$.

As a particular case, it is easily seen that

$$
D_{q}^{\alpha} x^{\mu-1}=\frac{\Gamma_{q}(\mu)}{\Gamma_{q}(\mu-\alpha)} x^{\mu-\alpha-1} .
$$

Next, we list some properties about $q$-derivative and $q$-integral that are already known in the literature.

Lemma 2.1 $([4,26])$ Let $\alpha, \beta \geq 0$ and $f$ be a function defined on $[0,1]$. Then the following formulas hold:

(i) $\left(I_{q}^{\beta} I_{q}^{\alpha} f\right)(x)=\left(I_{q}^{\alpha+\beta} f\right)(x)$,

(ii) $\left(D_{q}^{\alpha} I_{q}^{\alpha} f\right)=f(x)$.

Lemma 2.2 ([4]) Let $\alpha>0$ and $p$ be a positive integer. Then the following equality holds:

$$
\left(I_{q}^{\alpha} D_{q}^{p} f\right)(x)=\left(D_{q}^{p} I_{q}^{\alpha} f\right)(x)-\sum_{k=0}^{p-1} \frac{x^{\alpha-p+k}}{\Gamma_{q}(\alpha+k-p+1)}\left(D_{q}^{k} f\right)(0) .
$$

Lemma 2.3 ([25]) Let I and J be intervals containing zero such that $J \subseteq I$. Let $f_{n}$ and $f$ be functions defined in $I, n \in \mathbb{N}$ such that

$$
\lim _{n \rightarrow \infty} f_{n}(t)=f(t) \quad \text { for all } t \in I \text {, and } f_{n} \text { tends uniformly to } f \text { on } J .
$$

Then

$$
\lim _{n \rightarrow \infty} \int_{0}^{x} f_{n}(t) d_{q} t=\int_{0}^{x} f(t) d_{q} t \quad \text { for all } x \in I
$$

Lemma 2.4 ([28], Schauder fixed point theorem) Let $B$ be a Banach space with $C \subseteq B$ closed and convex. Assume that $U$ is a relatively open subset of $C$ with $0 \in U$ and $F: \bar{U} \rightarrow C$ is a continuous, compact map. Then either

(1) F has a fixed point in $\bar{U}$, or

(2) there exists $u \in \partial U$ and $\lambda \in(0,1)$ with $u=\lambda F u$.

Let $E=(E,\|\cdot\|)$ be a Banach space, $P \subset E$ be a cone, $\kappa$ be a nonnegative continuous concave functional on $P$, and $a, b, c>0$ be constants. Define $P_{c}=\{x \in P:\|x\|<c\}$, $P(\kappa, a, b)=\{x \in P: a \leq \kappa(x),\|x\|<b\}$. 
Lemma 2.5 (Leggett-Williams fixed point theorem) Let $T: \overline{P_{c}} \rightarrow \overline{P_{c}}$ be a completely continuous operator, and let $\kappa$ be a nonnegative continuous concave functional on $K$ such that $\kappa(x) \leq\|x\|$ for all $x \in \overline{P_{c}}$. Suppose that there exist $0<a<b<d \leq c$ such that

(A1) $\{x \in P(\kappa, b, d) \mid \kappa(x)>b\} \neq \emptyset$ and $\kappa(x)>b$ for $x \in P(\kappa, b, d)$;

(A2) $\|T x\|<a$ for $\|x\| \leq a$;

(A3) $\kappa(T x)>$ bor $x \in P(\kappa, b, c)$ with $\|T x\|>d$.

Then $T$ has at least three fixed points $x_{1}, x_{2}$, and $x_{3}$ such that $\left\|x_{1}\right\|<a, b<\kappa\left(x_{2}\right)$, and $\left\|x_{3}\right\|>a$, with $\kappa\left(x_{3}\right)<b$.

Remark 2.1 If there holds $d=c$, then condition (A1) implies condition (A3) in Lemma 2.5.

The next result is important in the sequel.

Lemma 2.6 Let $h \in C\left(\mathbb{R}^{+}\right)$be a given function. Then the boundary value problem

$$
\begin{aligned}
& \left(D_{q}^{\alpha} u\right)(t)+h(t)=0, \quad 0 \leq t<\infty, \\
& u(0)=0, \quad D_{q}^{\alpha-1} u(+\infty)=\sum_{i=1}^{m} a_{i} u\left(\xi_{i}\right),
\end{aligned}
$$

has a unique solution

$$
u(t)=\int_{0}^{\infty} G(t, q s) h(s) d_{q} s+\frac{t^{\alpha-1}}{\Gamma_{q}(\alpha)-\Delta} \sum_{i=1}^{m} a_{i} \int_{0}^{\infty} G\left(\xi_{i}, q s\right) h(s) d_{q} s,
$$

where

$$
\Delta=\sum_{i=1}^{m} a_{i} \xi_{i}^{\alpha-1}
$$

and

$$
G(t, q s)= \begin{cases}\frac{t^{\alpha-1}-(t-q s)(\alpha-1)}{\Gamma_{q}(\alpha)}, & 0 \leq s \leq t<\infty, \\ \frac{t^{\alpha-1}}{\Gamma_{q}(\alpha)}, & 0 \leq t \leq s<\infty,\end{cases}
$$

is the Green function of boundary value problem (2.2)-(2.3).

Proof Let us begin with integrating on both sides of (2.2)

$$
I_{q}^{\alpha} D_{q}^{\alpha} u(t)=-I_{q}^{\alpha} h(t)
$$

In view of Definition 2.2 and Lemma 2.2, we deduce

$$
u(t)=-\frac{1}{\Gamma_{q}(\alpha)} \int_{0}^{t}(t-q s)^{(\alpha-1)} h(s) d_{q} s+c_{1} t^{\alpha-1}+c_{2} t^{\alpha-2} .
$$

Applying the boundary condition $u(0)=0$, thus $c_{2}=0$, we have

$$
u(t)=-\frac{1}{\Gamma_{q}(\alpha)} \int_{0}^{t}(t-q s)^{(\alpha-1)} h(s) d_{q} s+c_{1} t^{\alpha-1} .
$$


Ma et al. Boundary Value Problems

(2019) 2019:46

Page 5 of 16

So,

$$
u\left(\xi_{i}\right)=c_{1} \xi_{i}^{\alpha-1}-\frac{1}{\Gamma_{q}(\alpha)} \int_{0}^{\xi_{i}}\left(\xi_{i}-q s\right)^{(\alpha-1)} h(s) d_{q} s,
$$

and

$$
\sum_{i=1}^{m} a_{i} u\left(\xi_{i}\right)=c_{1} \sum_{i=1}^{m} a_{i} \xi_{i}^{\alpha-1}-\frac{1}{\Gamma_{q}(\alpha)} \sum_{i=1}^{m} a_{i} \int_{0}^{\xi_{i}}\left(\xi_{i}-q s\right)^{(\alpha-1)} h(s) d_{q} s .
$$

Differentiating on both sides of (2.4) and combining (2.1), we get

$$
\begin{aligned}
& D_{q}^{\alpha-1} u(t) \\
& \quad=-D_{q}^{\alpha-1} I_{q}^{\alpha} h(t)+D_{q}^{\alpha-1} c_{1} t^{\alpha-1}=-D_{q} I_{q}^{1-(\alpha-1)} I_{q}^{\alpha} h(t)+c_{1} \frac{\Gamma_{q}(\alpha)}{\Gamma_{q}(\alpha-\alpha+1)} t^{\alpha-(\alpha-1)-1} \\
& \quad=-D_{q} I_{q}^{2-\alpha} I_{q}^{\alpha} h(t)+c_{1} \Gamma_{q}(\alpha)=-D_{q} I_{q}^{2} h(t)+c_{1} \Gamma_{q}(\alpha)=-I_{q} h(t)+c_{1} \Gamma_{q}(\alpha) .
\end{aligned}
$$

Therefore,

$$
D_{q}^{\alpha-1} u(\infty)=-\int_{0}^{+\infty} h(s) d_{q} s+c_{1} \Gamma_{q}(\alpha) .
$$

Using the boundary condition $D_{q}^{\alpha-1} u(+\infty)=\sum_{i=1}^{\infty} a_{i} u\left(\xi_{i}\right)$, hence

$$
-\int_{0}^{+\infty} h(s) d_{q} s+c_{1} \Gamma_{q}(\alpha)=c_{1} \sum_{i=1}^{m} a_{i} \xi_{i}^{\alpha-1}-\frac{1}{\Gamma_{q}(\alpha)} \sum_{i=1}^{m} a_{i} \int_{0}^{\xi_{i}}\left(\xi_{i}-q s\right)^{(\alpha-1)} h(s) d_{q} s .
$$

It is easy to show that

$$
\begin{aligned}
c_{1}= & \frac{1}{\Gamma_{q}(\alpha)-\sum_{i=1}^{m} a_{i} \xi_{i}} \int_{0}^{+\infty} h(s) d_{q} s \\
& -\frac{1}{\Gamma_{q}(\alpha)-\sum_{i=1}^{m} a_{i} \xi_{i}} \sum_{i=1}^{m} a_{i} \int_{0}^{\xi_{i}} \frac{\left(\xi_{i}-q s\right)^{(\alpha-1)}}{\Gamma_{q}(\alpha)} h(s) d_{q} s .
\end{aligned}
$$

For convenience, we denote

$$
\Delta=\sum_{i=1}^{m} a_{i} \xi_{i}^{\alpha-1}
$$

Utilizing (2.4) and (2.5), we get

$$
\begin{aligned}
u(t)= & \frac{t^{\alpha-1}}{\Gamma_{q}(\alpha)-\Delta} \int_{0}^{+\infty} h(s) d_{q} s-\frac{t^{\alpha-1}}{\Gamma_{q}(\alpha)-\Delta} \sum_{i=1}^{m} a_{i} \int_{0}^{\xi_{i}} \frac{\left(\xi_{i}-q s\right)^{(\alpha-1)}}{\Gamma_{q}(\alpha)} h(s) d_{q} s \\
& -\frac{1}{\Gamma_{q}(\alpha)} \int_{0}^{t}(t-q s)^{(\alpha-1)} h(s) d_{q} s \\
= & \left(\frac{1}{\Gamma_{q}(\alpha)}+\frac{\Delta}{\Gamma_{q}(\alpha)\left(\Gamma_{q}(\alpha)-\Delta\right)}\right) t^{\alpha-1} \int_{0}^{+\infty} h(s) d_{q} s
\end{aligned}
$$




$$
\begin{aligned}
& -\frac{t^{\alpha-1}}{\Gamma_{q}(\alpha)-\Delta} \sum_{i=1}^{m} a_{i} \int_{0}^{\xi_{i}} \frac{\left(\xi_{i}-q s\right)^{(\alpha-1)}}{\Gamma_{q}(\alpha)} h(s) d_{q} s \\
& -\frac{1}{\Gamma_{q}(\alpha)} \int_{0}^{t}(t-q s)^{(\alpha-1)} h(s) d_{q} s \\
= & \frac{1}{\Gamma_{q}(\alpha)} t^{\alpha-1} \int_{0}^{+\infty} h(s) d_{q} s-\frac{1}{\Gamma_{q}(\alpha)} \int_{0}^{t}(t-q s)^{(\alpha-1)} h(s) d_{q} s \\
& +\frac{\Delta}{\Gamma_{q}(\alpha)\left(\Gamma_{q}(\alpha)-\Delta\right)} t^{\alpha-1} \int_{0}^{+\infty} h(s) d_{q} s \\
& -\frac{t^{\alpha-1}}{\Gamma_{q}(\alpha)-\Delta} \sum_{i=1}^{m} a_{i} \int_{0}^{\xi_{i}} \frac{\left(\xi_{i}-q s\right)^{(\alpha-1)}}{\Gamma_{q}(\alpha)} h(s) d_{q} s \\
= & \int_{0}^{\infty} G(t, q s) h(s) d_{q} s+\frac{t^{\alpha-1}}{\Gamma_{q}(\alpha)-\Delta} \sum_{i=1}^{m} a_{i} \int_{0}^{\infty} G\left(\xi_{i}, q s\right) h(s) d_{q} s .
\end{aligned}
$$

Define

$$
G(t, q s)= \begin{cases}\frac{t^{\alpha-1}-(t-q)(\alpha-1)}{\Gamma_{q}(\alpha)}, & 0 \leq s \leq t<\infty, \\ \frac{t^{\alpha-1}}{\Gamma_{q}(\alpha)}, & 0 \leq t \leq s<\infty .\end{cases}
$$

The proof is completed.

The following properties of the Green function play important roles in this paper.

Lemma 2.7 Function $G$ defined in Lemma 2.6 satisfies the following properties:

(P1) $G(t, q s) \geq 0$ and $G(t, q s) \leq G(q s, q s)$ for all $0 \leq s, t<\infty$.

(P2) There exists a function $\gamma \in C\left(\mathbb{R}^{+}\right)$such that

$$
\min _{k \leq t \leq l} G(t, q s) \geq \gamma(q s) \sup _{0 \leq t<\infty} G(t, q s)=\gamma(q s) G(q s, q s), \quad s \in(0,+\infty)
$$

where $0<k<l<\infty$ are constants.

Proof It is clear that $G(t, q s) \geq 0$ for $s, t \in[0,+\infty)$. The monotonicity of $G(t, q s)$ implies

$$
\sup _{0 \leq t<+\infty} G(t, q s)=G(q s, q s)=\frac{(q s)^{\alpha-1}}{\Gamma_{q}(\alpha)}, \quad s \in[0,+\infty)
$$

Now we consider the existence of $\gamma$. Setting $g_{1}(t, q s)=\frac{t^{\alpha-1}-(t-q s)^{(\alpha-1)}}{\Gamma_{q}(\alpha)}$ and $g_{2}(t, q s)=\frac{t^{\alpha-1}}{\Gamma_{q}(\alpha)}$, we have

$$
\begin{aligned}
\min _{k \leq t \leq l} G(t, q s) & = \begin{cases}g_{1}(l, q s), & s \in(0, k], \\
\min \left\{g_{1}(l, q s), g_{2}(k, q s)\right\}, & s \in[k, l], \\
g_{2}(k, q s), & s \in[l, \infty)\end{cases} \\
& = \begin{cases}\frac{l^{\alpha-1}-(l-q s)}{\Gamma_{q}(\alpha-1)}, & s \in(0, r], \\
\frac{k^{\alpha-1}}{\Gamma_{q}(\alpha)}, & s \in[r, \infty),\end{cases}
\end{aligned}
$$


where $0<r<\infty$ is the unique solution of the equation

$$
l^{\alpha-1}-(l-q s)^{(\alpha-1)}=k^{\alpha-1} .
$$

Hence

$$
r(q s)= \begin{cases}\frac{l^{\alpha-1}-(l-q s)^{(\alpha-1)}}{(q s)^{\alpha-1}}, & 0<s \leq r \\ \left(\frac{k}{q s}\right)^{\alpha-1}, & r \leq s<\infty .\end{cases}
$$

This completes the proof of Lemma 2.7 .

\section{Main results}

We are now in a position to state and prove our main results in this paper.

Consider the space $C_{\infty}([0, \infty), \mathbb{R})$ defined by $C_{\infty}([0, \infty), \mathbb{R})=\{u \in C([0, \infty), \mathbb{R})$ : $\lim _{t \rightarrow+\infty} \frac{u(t)}{1+t^{\alpha-1}}$ exists $\}$ with the norm

$$
\|u\|=\sup _{t \in[0, \infty)} \frac{|u(t)|}{1+t^{\alpha-1}}
$$

Lemma 3.1 ([29]) $C_{\infty}$ is a Banach space.

For $u \in C_{\infty}$, we define the operator $T$ by

$$
T u(t)=\int_{0}^{\infty} G(t, q s) f(s, u(s)) d_{q} s+\frac{t^{\alpha-1}}{\Gamma_{q}(\alpha)-\Delta} \sum_{i=1}^{m} a_{i} \int_{0}^{\infty} G\left(\xi_{i}, q s\right) f(s, u(s)) d_{q} s
$$

We list some conditions in this section for convenience:

(H1) Let $F(t, u)=f\left(t,\left(1+t^{\alpha-1}\right) u\right),|F(t, u)| \leq \varphi(t) \omega(|u|)$ on $[0, \infty)$ with $\omega \in C([0, \infty), \mathbb{R})$ nondecreasing and $\varphi \in C([0, \infty)), \int_{0}^{\infty} \varphi(s) d_{q} s<+\infty$.

(H2) There exists a constant $\rho$ such that, for any $t \in[0, \infty), v_{1}, v_{2} \in \mathbb{R}$,

$$
\left|F\left(s, v_{2}(s)\right)-F\left(s, v_{1}(s)\right)\right| \leq \rho\left|v_{2}-v_{1}\right| .
$$

Since the Arzela-Ascoli theorem fails to work in the space $C_{\infty}$, we need a modified compactness criterion to prove $T$ is compact.

Lemma 3.2 ([29]) Let $V=\left\{u \in C_{\infty}:\|u\|<l\right\}(l>0), V_{1}=\left\{\frac{u(t)}{1+t^{\alpha-1}}: u \in V\right\}$. If $V_{1}$ is equicontinuous on any compact intervals of $[0,+\infty)$ and equiconvergent at infinity, then $V$ is relatively compact on $C_{\infty}$.

Remark 3.1 $V_{1}$ is called equiconvergent at infinity if and only if, for any given $\varepsilon>0$, there exists $N=N(\varepsilon)>0$ such that

$$
\left|\frac{u\left(t_{1}\right)}{1+t_{1}^{\alpha-1}}-\frac{u\left(t_{2}\right)}{1+t_{2}^{\alpha-1}}\right|<\varepsilon \quad \text { for all } u \in V_{1}, t_{1}, t_{2} \geq N .
$$

Define the cone $P \subset C_{\infty}$ by

$$
P=\left\{u \in C_{\infty}: u(t) \geq 0, t \in[0,+\infty)\right\} .
$$


Lemma 3.3 If $(\mathrm{H} 1)-(\mathrm{H} 2)$ hold, then $T: P \rightarrow P$ is completely continuous.

Proof We divide the proof into three steps.

Step 1: We show that $T: P \rightarrow P$ is continuous.

In view of the continuity and nonnegativity of $G$ and $f$, we have $T u(t) \geq 0$ for $t \in[0, \infty)$.

For any $u \in \Omega$, by (H1) we obtain

$$
\int_{0}^{\infty} f(s, u(s)) d_{q} s \leq \omega(L) \int_{0}^{\infty} \varphi(s) d_{q} s<\infty
$$

and

$$
\lim _{t \rightarrow+\infty} \frac{(T u)(t)}{1+t^{\alpha-1}}=\int_{0}^{\infty} \frac{1}{\Gamma_{q}(\alpha)} f(s, u(s)) d_{q} s+\frac{1}{\Gamma_{q}(\alpha)-\Delta} \sum_{i=1}^{m} a_{i} \int_{0}^{\infty} G\left(\xi_{i}, q s\right) f(s, u(s)) d_{q} s
$$

So, $\lim _{t \rightarrow+\infty} \frac{(T u)(t)}{1+t^{\alpha-1}}$ exists. Thus, $T(P) \subset P$.

Let $v(t)=\frac{u(t)}{1+t^{\alpha-1}}$. Then by $(\mathrm{H} 1)$, we have $f(s, u(s))=F\left(s, \frac{u_{n}(s)}{1+s^{\alpha-1}}\right)=F(s, v(s))$. Taking $u_{n} \rightarrow u$ as $n \rightarrow+\infty$ in $C_{\infty}$, by (H2) we deduce

$$
\begin{aligned}
\left|f\left(t, u_{n}(t)\right)-f(t, u(t))\right| & =\left|F\left(t, \frac{u_{n}(t)}{1+s^{\alpha-1}}\right)-F\left(t, \frac{u(t)}{1+t^{\alpha-1}}\right)\right|=\left|F\left(t, v_{n}(t)\right)-F(t, v(t))\right| \\
& \leq \rho\left|v_{n}(t)-v(t)\right| \rightarrow 0 \quad \text { uniformly on }[0, \infty) .
\end{aligned}
$$

So $f\left(t, u_{n}(t)\right) \rightarrow f(t, u(t))$ uniformly on $[0, \infty)$. By Lemma 2.3 we have

$$
\lim _{n \rightarrow \infty} \int_{0}^{\infty} f\left(s, u_{n}(s)\right) d_{q} s=\int_{0}^{\infty} f(s, u(s)) d_{q} s
$$

Then, combining (3.1), we have

$$
\begin{aligned}
\mid \frac{T u_{n}(t)}{1+} t^{\alpha-1} & \frac{T u(t)}{1+t^{\alpha-1}} \mid \\
= & \mid \int_{0}^{\infty} \frac{G(t, q s)}{1+t^{\alpha-1}}\left(f\left(s, u_{n}(s)\right)-f(s, u(s))\right) d_{q} s \\
& +\frac{t^{\alpha-1}}{\Gamma_{q}(\alpha)-\Delta} \sum_{i=1}^{m} a_{i} \int_{0}^{\infty} \frac{G\left(\xi_{i}, q s\right)}{1+t^{\alpha-1}}\left(f\left(s, u_{n}(s)\right)-f(s, u(s))\right) d_{q} s \mid \\
\leq & \left|\int_{0}^{\infty} \frac{G(t, q s)}{1+t^{\alpha-1}}\left(f\left(s, u_{n}(s)\right)-f(s, u(s))\right) d_{q} s\right| \\
& +\left|\frac{t^{\alpha-1}}{\Gamma_{q}(\alpha)-\Delta} \sum_{i=1}^{m} a_{i} \int_{0}^{\infty} \frac{G\left(\xi_{i}, q s\right)}{1+t^{\alpha-1}}\left(f\left(s, u_{n}(s)\right)-f(s, u(s))\right) d_{q} s\right| \\
\leq & \left|\frac{1}{\Gamma_{q}(\alpha)} \int_{0}^{\infty}\left(f\left(s, u_{n}(s)\right)-f(s, u(s))\right) d_{q} s\right| \\
& +\left|\frac{\sum_{i=1}^{m} a_{i} \xi_{i}^{\alpha-1}}{\Gamma_{q}(\alpha)\left(\Gamma_{q}(\alpha)-\Delta\right)} \int_{0}^{\infty}\left(f\left(s, u_{n}(s)\right)-f(s, u(s))\right) d_{q} s\right| .
\end{aligned}
$$


Hence

$$
\left\|T u_{n}-T u\right\|=\sup _{t \in[0, \infty)}\left|\frac{T u_{n}(t)-T u(t)}{1+t^{\alpha-1}}\right| \rightarrow 0, \quad \text { as } n \rightarrow \infty
$$

So, $T$ is continuous.

Step 2: We show that $T$ is uniformly bounded.

Let $\Omega$ be any bounded subset of $P$, i.e., there exists $L>0$ such that $\|u\| \leq L$ for each $u \in \Omega$. It suffices to show that $T(u)$ is bounded in $P$. In fact, for $u \in \Omega$, we have

$$
\begin{aligned}
\|T u\|= & \sup _{t \in[0, \infty)}\left(\int_{0}^{\infty} \frac{G(t, q s)}{1+t^{\alpha-1}} f(t, u(s)) d_{q} s\right. \\
& \left.+\frac{t^{\alpha-1}}{\Gamma_{q}(\alpha)-\Delta} \sum_{i=1}^{m} a_{i} \int_{0}^{\infty} \frac{G\left(\xi_{i}, q s\right)}{1+t^{\alpha-1}} f(t, u(s)) d_{q} s\right) \\
\leq & \int_{0}^{\infty} \frac{1}{\Gamma_{q}(\alpha)}|f(t, u(s))| d_{q} s+\frac{\sum_{i=1}^{m} a_{i} \xi_{i}^{\alpha-1}}{\Gamma_{q}(\alpha)-\Delta} \int_{0}^{\infty} \frac{1}{\Gamma_{q}(\alpha)}|f(t, u(s))| d_{q} s \\
= & \left(\frac{1}{\Gamma_{q}(\alpha)}+\frac{\sum_{i=1}^{m} a_{i} \xi_{i}^{\alpha-1}}{\left(\Gamma_{q}(\alpha)-\Delta\right) \Gamma_{q}(\alpha)}\right) \int_{0}^{\infty}\left|f\left(s, \frac{u(s)\left(1+s^{\alpha-1}\right)}{1+s^{\alpha-1}}\right)\right| d_{q} s \\
= & \left(\frac{1}{\Gamma_{q}(\alpha)}+\frac{\Delta}{\left(\Gamma_{q}(\alpha)-\Delta\right) \Gamma_{q}(\alpha)}\right) \int_{0}^{\infty}\left|F\left(s, \frac{u(s)}{1+s^{\alpha-1}}\right)\right| d_{q} s \\
\leq & \frac{\omega(L)}{\Gamma_{q}(\alpha)-\Delta} \int_{0}^{\infty} \varphi(s) d_{q} s<\infty .
\end{aligned}
$$

Hence, $T(\Omega)$ is uniformly bounded.

Now, we show that $T(\Omega)$ is equicontinuous on any compact interval.

First, for any given $Q>0, t_{1}, t_{2} \in[0, Q]$, and $u \in \Omega$, without loss of generality, we assume that $t_{2}>t_{1}$, we deduce

$$
\begin{aligned}
& \left|\frac{T u\left(t_{2}\right)}{1+} t_{2}^{\alpha-1}-\frac{T u\left(t_{1}\right)}{1+t_{1}^{\alpha-1}}\right| \\
& \leq\left|\int_{0}^{\infty} \frac{G\left(t_{2}, q s\right)}{1+t_{2}^{\alpha-1}} f(s, u(s)) d_{q} s-\int_{0}^{\infty} \frac{G\left(t_{1}, q s\right)}{1+t_{1}^{\alpha-1}} f(s, u(s)) d_{q} s\right| \\
& \quad+\left|\left(\frac{t_{2}^{\alpha-1}}{1+t_{2}^{\alpha-1}}-\frac{t_{1}^{\alpha-1}}{1+t_{1}^{\alpha-1}}\right) \sum_{i=1}^{m} a_{i} \int_{0}^{\infty} \frac{G\left(\xi_{i}, q s\right)}{\Gamma_{q}(\alpha)-\Delta} f(s, u(s)) d_{q} s\right| \\
& \leq\left|\int_{0}^{\infty} \frac{G\left(t_{2}, q s\right)}{1+t_{2}^{\alpha-1}} f(s, u(s)) d_{q} s-\int_{0}^{\infty} \frac{G\left(t_{1}, q s\right)}{1+t_{2}^{\alpha-1}} f(s, u(s)) d_{q} s\right| \\
& \quad+\left|\int_{0}^{\infty} \frac{G\left(t_{1}, q s\right)}{1+t_{2}^{\alpha-1}} f(s, u(s)) d_{q} s-\int_{0}^{\infty} \frac{G\left(t_{1}, q s\right)}{1+t_{1}^{\alpha-1}} f(s, u(s)) d_{q} s\right| \\
& \quad+\left|\left(\frac{t_{2}^{\alpha-1}}{1+t_{2}^{\alpha-1}}-\frac{t_{1}^{\alpha-1}}{1+t_{1}^{\alpha-1}}\right) \sum_{i=1}^{m} a_{i} \int_{0}^{\infty} \frac{G\left(\xi_{i}, q s\right)}{\Gamma_{q}(\alpha)-\Delta} f(s, u(s)) d_{q} s\right| .
\end{aligned}
$$


On the other hand, for all $u \in \Omega, t_{1} \rightarrow t_{2}$, we have that

$$
\begin{aligned}
\left|\int_{0}^{\infty} \frac{G\left(t_{2}, q s\right)}{1+t_{2}^{\alpha-1}} f(s, u(s)) d_{q} s-\int_{0}^{\infty} \frac{G\left(t_{1}, q s\right)}{1+t_{2}^{\alpha-1}} f(s, u(s)) d_{q} s\right| & \int_{0}^{t_{1}}\left|\frac{\left(t_{2}^{\alpha-1}-t_{1}^{\alpha-1}\right)-\left(\left(t_{2}-q s\right)^{(\alpha-1)}-\left(t_{1}-q s\right)^{(\alpha-1)}\right)}{\Gamma_{q}(\alpha)\left(1+t_{2}^{\alpha-1}\right)}\right| f(s, u(s)) d_{q} s \\
\leq & +\int_{t_{1}}^{t_{2}}\left|\frac{t_{2}^{\alpha-1}-t_{1}^{\alpha-1}-\left(t_{2}-q s\right)^{(\alpha-1)}}{\Gamma_{q}(\alpha)\left(1+t_{2}^{\alpha-1}\right)}\right| f(s, u(s)) d_{q} s \\
& +\int_{t_{2}}^{\infty}\left|\frac{t_{2}^{\alpha-1}-t_{1}^{\alpha-1}}{\Gamma_{q}(\alpha)\left(1+t_{2}^{\alpha-1}\right)}\right| f(s, u(s)) d_{q} s \\
\leq & \int_{0}^{t_{1}} \frac{\left(t_{2}^{\alpha-1}-t_{1}^{\alpha-1}\right)+\left(\left(t_{2}-q s\right)^{(\alpha-1)}-\left(t_{1}-q s\right)^{(\alpha-1)}\right)}{\Gamma_{q}(\alpha)\left(1+t_{2}^{\alpha-1}\right)} f(s, u(s)) d_{q} s \\
& +\frac{3 Q^{\alpha-1}}{\Gamma_{q}(\alpha)\left(1+t_{2}^{\alpha-1}\right)} \int_{t_{1}}^{t_{2}} f(s, u(s)) d_{q} s+\frac{t_{2}^{\alpha-1}-t_{1}^{\alpha-1}}{\Gamma_{q}(\alpha)\left(1+t_{2}^{\alpha-1}\right)} \int_{t_{2}}^{\infty} f(s, u(s)) d_{q} s \\
\leq & \omega(L) \int_{0}^{t_{1}} \frac{\left(t_{2}^{\alpha-1}-t_{1}^{\alpha-1}\right)+\left(\left(t_{2}-q s\right)^{(\alpha-1)}-\left(t_{1}-q s\right)^{(\alpha-1)}\right)}{\Gamma_{q}(\alpha)\left(1+t_{2}^{\alpha-1}\right)} \varphi(s) d_{q} s \\
& +\omega(L) \frac{3 Q^{\alpha-1}}{\Gamma_{q}(\alpha)\left(1+t_{2}^{\alpha-1}\right)} \int_{t_{1}}^{t_{2}} \varphi(s) d_{q} s+\omega(L) \frac{t_{2}^{\alpha-1}-t_{1}^{\alpha-1}}{\Gamma_{q}(\alpha)\left(1+t_{2}^{\alpha-1}\right)} \int_{t_{2}}^{\infty} \varphi(s) d_{q} s \\
\rightarrow & 0 .
\end{aligned}
$$

Similar to (3.2), for all $u \in \Omega, t_{1} \rightarrow t_{2}$, we have

$$
\begin{aligned}
& \left|\int_{0}^{\infty} \frac{G\left(t_{1}, q s\right)}{1+t_{2}^{\alpha-1}} f(s, u(s)) d_{q} s-\int_{0}^{\infty} \frac{G\left(t_{1}, q s\right)}{1+t_{1}^{\alpha-1}} f(s, u(s)) d_{q} s\right| \\
& \quad \leq \int_{0}^{\infty} \frac{G_{1}(t, q s)\left|\left(1+t_{1}^{\alpha-1}\right)-\left(1+t_{2}^{\alpha-1}\right)\right|}{\left(1+t_{1}^{\alpha-1}\right)\left(1+t_{2}^{\alpha-1}\right)} f(s, u(s)) d_{q} s \\
& \quad \leq \frac{\left|t_{2}^{\alpha-1}-t_{1}^{\alpha-1}\right|}{\left(1+t_{2}^{\alpha-1}\right)} \int_{0}^{\infty} \frac{G\left(t_{1}, q s\right)}{1+t_{1}^{\alpha-1}} f(s, u(s)) d_{q} s \\
& \quad \leq \frac{\left|t_{2}^{\alpha-1}-t_{1}^{\alpha-1}\right|}{\left(1+t_{2}^{\alpha-1}\right)} \int_{0}^{\infty} \frac{1}{\Gamma_{q}(\alpha)} \omega(L) \varphi(s) d_{q} s \leq \omega(L) \frac{\left|t_{2}^{\alpha-1}-t_{1}^{\alpha-1}\right|}{\Gamma_{q}(\alpha)\left(1+t_{2}^{\alpha-1}\right)} \int_{0}^{\infty} \varphi(s) d_{q} s \rightarrow 0,
\end{aligned}
$$

and

$$
\begin{aligned}
& \left|\left(\frac{t_{2}^{\alpha-1}}{1+t_{2}^{\alpha-1}}-\frac{t_{1}^{\alpha-1}}{1+t_{1}^{\alpha-1}}\right) \sum_{i=1}^{m} a_{i} \int_{0}^{\infty} \frac{G\left(\xi_{i}, q s\right)}{\Gamma_{q}(\alpha)-\Delta} f(s, u(s)) d_{q} s\right| \\
& \quad \leq \frac{\left|t_{2}^{\alpha-1}-t_{1}^{\alpha-1}\right|}{\left(1+t_{2}^{\alpha-1}\right)\left(1+t_{1}^{\alpha-1}\right)} \int_{0}^{\infty} \frac{\sum_{i=1}^{m} a_{i} \xi_{i}^{\alpha-1}}{\Gamma_{q}(\alpha-\Delta) \Gamma_{q}(\alpha)} f(s, u(s)) d_{q} s \\
& \quad \leq \frac{\left|t_{2}^{\alpha-1}-t_{1}^{\alpha-1}\right|}{\left(1+t_{2}^{\alpha-1}\right)\left(1+t_{1}^{\alpha-1}\right)} \frac{\omega(L) \Delta}{\Gamma_{q}(\alpha-\Delta) \Gamma_{q}(\alpha)} \int_{0}^{\infty} \varphi(s) d_{q} s \rightarrow 0 .
\end{aligned}
$$

Hence $T(\Omega)$ is equicontinuous on any compact intervals of $[0, \infty)$.

Step 3: We show that $T$ is equiconvergent at $\infty$. For any $u \in \Omega$, by $(\mathrm{H} 1)$

$$
\int_{0}^{\infty} f(s, u(s)) d_{q} s \leq \omega(L) \int_{0}^{\infty} \varphi(s) d_{q} s<\infty
$$


On the other hand, since $\lim _{t \rightarrow \infty} \frac{t^{\alpha-1}}{1+t^{\alpha-1}}=1$, there exists $T_{1}>0$ such that, for any $t_{2}>t_{1}>$ $T_{1}$,

$$
\left|\frac{t_{2}^{\alpha-1}}{1+t_{2}^{\alpha-1}}-\frac{t_{1}^{\alpha-1}}{1+t_{1}^{\alpha-1}}\right| \leq\left|1-\frac{t_{2}^{\alpha-1}}{1+t_{2}^{\alpha-1}}\right|+\left|1-\frac{t_{1}^{\alpha-1}}{1+t_{1}^{\alpha-1}}\right|<\varepsilon
$$

Similarly, there exists a constant $M>0$ such that $\lim _{t \rightarrow \infty} \frac{(t-M)^{(\alpha-1)}}{1+t^{\alpha-1}}=1$ and there exists $T_{2}$ such that, for any $t_{2}>t_{1}>T_{2}$ and $0 \leq s \leq M$,

$$
\begin{aligned}
\left|\frac{\left(t_{2}-s\right)^{(\alpha-1)}}{1+t_{2}^{\alpha-1}}-\frac{\left(t_{1}-s\right)^{(\alpha-1)}}{1+t_{1}^{\alpha-1}}\right| & \leq\left|1-\frac{\left(t_{2}-s\right)^{(\alpha-1)}}{1+t_{2}^{\alpha-1}}\right|+\left|1-\frac{\left(t_{1}-s\right)^{(\alpha-1)}}{1+t_{1}^{\alpha-1}}\right| \\
& \leq\left|1-\frac{\left(t_{2}-M\right)^{(\alpha-1)}}{1+t_{2}^{\alpha-1}}\right|+\left|1-\frac{\left(t_{1}-M\right)^{(\alpha-1)}}{1+t_{1}^{\alpha-1}}\right|<\varepsilon .
\end{aligned}
$$

Choose $N>\max \left\{T_{1}, T_{2}\right\}$, then for any $u \in \Omega, t_{2}>t_{1}>N$ and $t_{1} \rightarrow t_{2}$, by (3.4)-(3.5), we have

$$
\begin{aligned}
& \left|\int_{0}^{\infty} \frac{G\left(t_{2}, q s\right)}{1+t_{2}^{\alpha-1}} f(s, u(s)) d_{q} s-\int_{0}^{\infty} \frac{G\left(t_{1}, q s\right)}{1+t_{1}^{\alpha-1}} f(s, u(s)) d_{q} s\right| \\
& \leq \frac{1}{\Gamma_{q}(\alpha)} \int_{0}^{t_{1}}\left|\frac{t_{2}^{\alpha-1}-\left(t_{2}-q s\right)^{(\alpha-1)}}{1+t_{2}^{\alpha-1}}-\frac{t_{1}^{\alpha-1}-\left(t_{1}-q s\right)^{(\alpha-1)}}{1+t_{1}^{\alpha-1}}\right| f(s, u(s)) d_{q} s \\
& +\frac{1}{\Gamma_{q}(\alpha)} \int_{t_{1}}^{t_{2}}\left|\frac{t_{2}^{\alpha-1}-\left(t_{2}-q s\right)^{(\alpha-1)}}{1+t_{2}^{\alpha-1}}-\frac{t_{1}^{\alpha-1}}{1+t_{1}^{\alpha-1}}\right| f(s, u(s)) d_{q} s \\
& +\frac{1}{\Gamma_{q}(\alpha)} \int_{t_{2}}^{\infty}\left|\frac{t_{2}^{\alpha-1}}{1+t_{2}^{\alpha-1}}-\frac{t_{1}^{\alpha-1}}{1+t_{1}^{\alpha-1}}\right| f(s, u(s)) d_{q} s \\
& \leq \frac{1}{\Gamma_{q}(\alpha)} \int_{0}^{t_{1}}\left|\frac{t_{2}^{\alpha-1}}{1+t_{2}^{\alpha-1}}-\frac{t_{1}^{\alpha-1}}{1+t_{1}^{\alpha-1}}-\frac{\left(t_{2}-q s\right)^{(\alpha-1)}}{1+t_{2}^{\alpha-1}}+\frac{\left(t_{1}-q s\right)^{(\alpha-1)}}{1+t_{1}^{\alpha-1}}\right| f(s, u(s)) d_{q} s \\
& +\frac{1}{\Gamma_{q}(\alpha)} \int_{t_{1}}^{t_{2}}\left(\frac{t_{2}^{\alpha-1}}{1+t_{2}^{\alpha-1}}-\frac{t_{1}^{\alpha-1}}{1+t_{1}^{\alpha-1}}+\frac{\left(t_{2}-q s\right)^{(\alpha-1)}}{1+t_{2}^{\alpha-1}}\right) f(s, u(s)) d_{q} s \\
& +\frac{1}{\Gamma_{q}(\alpha)} \int_{t_{2}}^{\infty}\left|\frac{t_{2}^{\alpha-1}}{1+t_{2}^{\alpha-1}}-\frac{t_{1}^{\alpha-1}}{1+t_{1}^{\alpha-1}}\right| f(s, u(s)) d_{q} s \\
& \leq \frac{1}{\Gamma_{q}(\alpha)}\left(\int_{0}^{t_{1}} 2 \varepsilon f(s, u(s)) d_{q} s+\int_{t_{1}}^{t_{2}}(\varepsilon+1) f(s, u(s)) d_{q} s+\int_{t_{2}}^{\infty} \varepsilon f(s, u(s)) d_{q} s\right) \\
& \leq \frac{\varepsilon \omega(L)}{\Gamma_{q}(\alpha)}\left(\int_{0}^{t_{1}} \varphi(s) d_{q} s+\int_{0}^{\infty} \varphi(s) d_{q} s\right)+\omega(L) \int_{t_{1}}^{t_{2}} \varphi(s) d_{q} s \\
& \rightarrow 0 \text { uniformly as } t_{2}, t_{1}>N,
\end{aligned}
$$

and

$$
\begin{aligned}
& \left|\left(\frac{t_{2}^{\alpha-1}}{1+t_{2}^{\alpha-1}}-\frac{t_{1}^{\alpha-1}}{1+t_{1}^{\alpha-1}}\right) \sum_{i=1}^{m} a_{i} \int_{0}^{\infty} \frac{G\left(\xi_{i}, q s\right)}{\Gamma_{q}(\alpha)-\Delta} f(s, u(s)) d_{q} s\right| \\
& \quad \leq\left|\frac{t_{2}^{\alpha-1}}{1+t_{2}^{\alpha-1}}-\frac{t_{1}^{\alpha-1}}{1+t_{1}^{\alpha-1}}\right| \sum_{i=1}^{m} a_{i} \int_{0}^{\infty} \frac{\xi_{i}^{\alpha-1}}{\left(\Gamma_{q}(\alpha)-\Delta\right) \Gamma_{q}(\alpha)} f(s, u(s)) d_{q} s
\end{aligned}
$$




$$
\begin{aligned}
& \leq\left|\frac{t_{2}^{\alpha-1}}{1+t_{2}^{\alpha-1}}-\frac{t_{1}^{\alpha-1}}{1+t_{1}^{\alpha-1}}\right| \frac{\Delta \omega(L)}{\left(\Gamma_{q}(\alpha)-\Delta\right) \Gamma_{q}(\alpha)} \int_{0}^{\infty} \varphi(s) d_{q} s \\
& \rightarrow 0 \quad \text { uniformly as } t_{2}, t_{1}>N .
\end{aligned}
$$

It can be easily seen from (3.6) and (3.7) that, for any $\varepsilon>0$, there exists a sufficiently large $N>0$ such that, for any $u \in \Omega$,

$$
\left|\frac{(T u)\left(t_{1}\right)}{1+t_{1}^{\alpha-1}}-\frac{(T u)\left(t_{2}\right)}{1+t_{2}^{\alpha-1}}\right|<\varepsilon \quad \text { for all } t_{1}, t_{2} \geq N .
$$

This implies that $T: P \rightarrow P$ is equiconvergent at $\infty$.

By using Lemma 3.2, we obtain that $T: P \rightarrow P$ is completely continuous. This completes the proof.

Theorem 3.1 Assume that $(\mathrm{H} 1)-(\mathrm{H} 2)$ hold. There exists a constant $v>0$ such that $\omega$ and $\varphi$ satisfy the following condition:

$$
\frac{v\left(\Gamma_{q}(\alpha)-\Delta\right)}{\omega(v) \int_{0}^{\infty} \varphi(s) d_{q} s}>1
$$

Then boundary value problem (1.1)-(1.2) has an unbounded solution $u(t)$ such that

$$
0 \leq \frac{u(t)}{1+t^{\alpha-1}} \leq v \quad \text { for } t \in[0, \infty) .
$$

Proof Let

$$
U=\{u \in P:\|u\| \leq v\}
$$

We claim that $u \neq \lambda T u$ for $u \in \partial U$ and $\lambda \in(0,1)$. The claim is immediate, since if there exists $u \in \partial U$ with $u=\lambda T u$, then for $\lambda \in(0,1)$, we have

$$
\begin{aligned}
\|u\|= & \sup _{t \in[0, \infty)}\left|\frac{\lambda T u(t)}{1+t^{\alpha-1}}\right| \leq \sup _{t \in[0, \infty)}\left|\frac{T u(t)}{1+t^{\alpha-1}}\right| \\
= & \sup _{t \in[0, \infty)}\left(\int_{0}^{\infty} \frac{G(t, q s)}{1+t^{\alpha-1}} f(s, u(s)) d_{q} s\right. \\
& \left.+\frac{t^{\alpha-1}}{\Gamma_{q}(\alpha)-\Delta} \sum_{i=1}^{m} a_{i} \int_{0}^{\infty} \frac{G\left(\xi_{i}, q s\right)}{1+t^{\alpha-1}} f(s, u(s)) d_{q} s\right) \\
\leq & \int_{0}^{\infty} \frac{1}{\Gamma_{q}(\alpha)}|f(s, u(s))| d_{q} s+\frac{\sum_{i=1}^{m} a_{i} \xi^{\alpha-1}}{\Gamma_{q}(\alpha)-\Delta} \int_{0}^{\infty} \frac{1}{\Gamma_{q}(\alpha)}|f(s, u(s))| d_{q} s \\
= & \left(\frac{1}{\Gamma_{q}(\alpha)}+\frac{\Delta}{\left(\Gamma_{q}(\alpha)-\Delta\right) \Gamma_{q}(\alpha)}\right) \int_{0}^{\infty}\left|f\left(s, \frac{u(s)\left(1+s^{\alpha-1}\right)}{1+s^{\alpha-1}}\right)\right| d_{q} s \\
= & \left(\frac{1}{\Gamma_{q}(\alpha)}+\frac{\Delta}{\left(\Gamma_{q}(\alpha)-\Delta\right) \Gamma_{q}(\alpha)}\right) \int_{0}^{\infty}\left|F\left(s, \frac{u(s)}{1+s^{\alpha-1}}\right)\right| d_{q} s \\
\leq & \frac{\omega(v)}{\Gamma_{q}(\alpha)-\Delta} \int_{0}^{\infty} \varphi(s) d_{q} s .
\end{aligned}
$$


So, for $u \in \partial U$, we obtain

$$
v \leq \frac{\omega(v)}{\Gamma_{q}(\alpha)-\Delta} \int_{0}^{\infty} \varphi(s) d_{q} s,
$$

that is,

$$
\frac{v\left(\Gamma_{q}(\alpha)-\Delta\right)}{\omega(v) \int_{0}^{\infty} \varphi(s) d_{q} s} \leq 1
$$

which contradicts with (3.8). By Lemma 2.4, boundary value problem (1.1)-(1.2) has an unbounded solution $u=u(t)$ such that

$$
0 \leq \frac{u(t)}{1+t^{\alpha-1}} \leq v \quad \text { for } t \in[0, \infty) .
$$

The proof is completed.

We define a nonnegative continuous concave functional $\kappa(u)=\min _{t \in[k, l]} \frac{|u(t)|}{1+t^{\alpha-1}}$ for $u \in P$, $k$, and $l$ is defined by (2.8). Denote

$$
N_{1}=\frac{\Gamma_{q}(\alpha)-\Delta}{\int_{0}^{\infty} \varphi(s) d_{q} s}, \quad N_{2}=\frac{\left(1+l^{\alpha-1}\right)\left(\Gamma_{q}(\alpha)-\Delta\right)}{\left(\Gamma_{q}(\alpha)-\Delta+\sum_{i=1}^{m} a_{i} k^{\alpha-1}\right) \int_{k}^{l} \gamma(q s) G(q s, q s) d_{q} s} .
$$

Theorem 3.2 Assume that there exist constants $a, b, c$ with $0<a<b<c$ such that $f(t, u)$ satisfies $(\mathrm{H} 1)-(\mathrm{H} 2)$ and the following conditions:

(H3) $\omega(u)<N_{1}$ a for all $u \in[0, a] ; \omega(u) \leq N_{1}$ c for all $u \in[0, c]$.

(H4) $F\left(t, \frac{u}{1+t^{\alpha-1}}\right)>N_{2} b$ for all $\left(t, \frac{u}{1+t^{\alpha-1}}\right) \in[k, l] \times[b, c]$.

Then the boundary value problem (1.1)-(1.2) has at least three solutions $u_{1}, u_{2}, u_{3}$ such that $\left\|u_{1}\right\|<a, b<\kappa\left(u_{2}(t)\right)$ and $\left\|u_{3}\right\|>a$ with $\kappa\left(u_{3}(t)\right)<b$ for $t \in[0, \infty)$.

Proof Firstly, we show that $T: \overline{P_{c}} \rightarrow \overline{P_{c}}$ is a completely continuous operator.

In fact, for $u \in \overline{P_{c}}$, then $\|u\| \leq c$, by (H1) and (H3), we get

$$
\begin{aligned}
\|T u\|= & \sup _{t \in[0, \infty)} \frac{T u(t)}{1+t^{\alpha-1}} \\
\leq & \int_{0}^{\infty} \frac{G(t, q s)}{1+t^{\alpha-1}}\left|f\left(s, \frac{u(s)\left(1+s^{\alpha-1}\right)}{1+s^{\alpha-1}}\right)\right| d_{q} s \\
& +\frac{t^{\alpha-1}}{\Gamma_{q}(\alpha)-\Delta} \sum_{i=1}^{m} a_{i} \int_{0}^{\infty} \frac{G\left(\xi_{i}, q s\right)}{1+t^{\alpha-1}}\left|f\left(s, \frac{u(s)\left(1+s^{\alpha-1}\right)}{1+s^{\alpha-1}}\right)\right| d_{q} s \\
\leq & \left(\frac{1}{\Gamma_{q}(\alpha)}+\frac{\Delta}{\Gamma_{q}(\alpha)\left(\Gamma_{q}(\alpha)-\Delta\right)}\right) \int_{0}^{\infty} F\left(s, \frac{u(s)}{1+s^{\alpha-1}}\right) d_{q} s \\
\leq & \frac{1}{\Gamma_{q}(\alpha)-\Delta} \int_{0}^{\infty} \varphi(s) \omega\left(\frac{|u|}{1+s^{\alpha-1}}\right) d_{q} s \\
\leq & \frac{1}{\Gamma_{q}(\alpha)-\Delta} \int_{0}^{\infty} \varphi(s) \omega(|u|) d_{q} s \leq \frac{N_{1} c}{\Gamma_{q}(\alpha)-\Delta} \int_{0}^{\infty} \varphi(s) d_{q} s \leq c .
\end{aligned}
$$

Hence, $\|T u\| \leq c$, that is, $T: \overline{P_{c}} \rightarrow \overline{P_{c}}$. In view of Lemma 3.3, $T: \overline{P_{c}} \rightarrow \overline{P_{c}}$ is completely continuous. 
In an analogous way, condition (H3) implies that condition (A2) of Lemma 2.5 is satisfied. Let $u(t)=\frac{(b+c)\left(1+t^{\alpha-1}\right)}{2}, 0 \leq t<\infty$. Then it is easy to see $\|u\|=\frac{(b+c)}{2}, u\left(\frac{(b+c)\left(1+t^{\alpha-1}\right)}{2}\right)=$ $\frac{(b+c)}{2} \in P(\kappa, b, c), \kappa\left(\frac{(b+c)\left(1+t^{\alpha-1}\right)}{2}\right)=\frac{(b+c)}{2}>b$, so $\{u \in P(\kappa, b, c) \mid \kappa(u)>b\} \neq \emptyset$. If $u \in P(\kappa, b, c)$, then $b \leq \frac{u(t)}{1+t^{\alpha-1}} \leq c$ for $t \in[k, l]$. By (H4) and Lemma 2.7, we get

$$
\begin{aligned}
& \kappa(u)=\min _{t \in[k, l]} \frac{|u(t)|}{1+t^{\alpha-1}} \\
& =\min _{t \in[k, l]} \mid \int_{0}^{\infty} \frac{G(t, q s)}{1+t^{\alpha-1}} f\left(s, \frac{u(s)\left(1+s^{\alpha-1}\right)}{1+s^{\alpha-1}}\right) d_{q} s \\
& +\frac{t^{\alpha-1}}{\Gamma_{q}(\alpha)-\Delta} \sum_{i=1}^{m} a_{i} \int_{0}^{\infty} \frac{G\left(\xi_{i}, q s\right)}{1+t^{\alpha-1}} f\left(s, \frac{u(s)\left(1+s^{\alpha-1}\right)}{1+s^{\alpha-1}}\right) d_{q} s \\
& \geq \int_{0}^{\infty} \frac{G(t, q s)}{1+l^{\alpha-1}} f\left(s, \frac{u(s)\left(1+s^{\alpha-1}\right)}{1+s^{\alpha-1}}\right) d_{q} s \\
& +\frac{k^{\alpha-1}}{\Gamma_{q}(\alpha)-\Delta} \sum_{i=1}^{m} a_{i} \int_{0}^{\infty} \frac{G\left(\xi_{i}, q s\right)}{1+l^{\alpha-1}} f\left(s, \frac{u(s)\left(1+s^{\alpha-1}\right)}{1+s^{\alpha-1}}\right) d_{q} s \\
& \geq \int_{0}^{\infty} \frac{\gamma(q s) G(q s, q s)}{1+l^{\alpha-1}} f\left(s, \frac{u(s)\left(1+s^{\alpha-1}\right)}{1+s^{\alpha-1}}\right) d_{q} s \\
& +\frac{k^{\alpha-1}}{\Gamma_{q}(\alpha)-\Delta} \sum_{i=1}^{m} a_{i} \int_{0}^{\infty} \frac{\gamma(q s) G(q s, q s)}{1+l^{\alpha-1}} f\left(s, \frac{u(s)\left(1+s^{\alpha-1}\right)}{1+s^{\alpha-1}}\right) d_{q} s \\
& =\left(\frac{1}{1+l^{\alpha-1}}+\frac{\sum_{i=1}^{m} a_{i} k^{\alpha-1}}{\Gamma_{q}(\alpha)-\Delta}\right) \int_{k}^{l} \gamma(q s) G(q s, q s) F\left(s, \frac{u(s)}{1+s^{\alpha-1}}\right) d_{q} s \\
& >\left(\frac{1}{1+l^{\alpha-1}}+\frac{\sum_{i=1}^{m} a_{i} k^{\alpha-1}}{\Gamma_{q}(\alpha)-\Delta}\right) \int_{k}^{l} \gamma(q s) G(q s, q s) N_{2} b d_{q} s=b .
\end{aligned}
$$

So condition (A1) of Lemma 2.5 is satisfied.

By Lemma 2.5 and Remark 2.1, there exist three solutions $u_{1}, u_{2}, u_{3}$ such that $\left\|u_{1}\right\|<a$, $b<\kappa\left(u_{2}(t)\right)$, and $\left\|u_{3}\right\|>a$ with $\kappa\left(u_{3}(t)\right)<b$, which completes the proof.

\section{Example}

In this section, we will give an example to expound our main results.

Example 4.1 Consider the following boundary value problem:

$$
\begin{aligned}
& \left(D_{q}^{\frac{3}{2}} u\right)(t)+f(t, u(t))=0, \quad t \geq 0, \\
& u(0)=0, \quad D_{q}^{\frac{1}{2}} u(+\infty)=\sum_{i=1}^{m} a_{i} u\left(\xi_{i}\right),
\end{aligned}
$$

here $\alpha=\frac{3}{2}, 0 \leq \Delta=\sum_{i=1}^{m} a_{i} \xi_{i}^{\alpha-1}<\Gamma_{q}\left(\frac{3}{2}\right), f(t, u)=\sqrt{\left|\frac{u}{1+t^{\frac{1}{2}}}\right|} e^{-t}, F(t, u)=\sqrt{|u|} e^{-t}$.

Choose $\omega(u)=\sqrt{u}, \varphi(t)=e^{-t}, k>\left(\frac{1}{\Gamma_{q}\left(\frac{3}{2}\right)-\Delta}\right)^{2}$, we have:

(i) $0 \leq \sum_{i=1}^{m} a_{i} \xi_{i}^{\alpha-1}<\Gamma_{q}(\alpha)$;

(ii) $f:[0,+\infty) \times \mathbb{R} \rightarrow[0,+\infty)$ is continuous; 
(iii) $|F(t, u)|=\varphi(t) \omega(|u|)$ on $[0, \infty) \times \mathbb{R}$ with $\omega \in C([0, \infty), \mathbb{R})$ nondecreasing and $\int_{0}^{\infty} \varphi(s) d_{q} s<+\infty$

(iv) $\frac{k\left(\Gamma_{q}(\alpha)-\Delta\right)}{\omega(k) \int_{0}^{\infty} \varphi(s) d_{q} s}=\sqrt{k}\left(\Gamma_{q}\left(\frac{3}{2}\right)-\Delta\right)>1$.

Thus, from Theorem 3.1, problem (4.1)-(4.2) has a positive solution $u$ such that

$$
0 \leq \frac{u(t)}{1+\sqrt{t}} \leq k \quad \text { for } t \in[0, \infty)
$$

\section{Acknowledgements}

The authors sincerely thank the reviewers for their valuable suggestions and useful comments that have led to the present improved version of the original manuscript.

\section{Funding}

This research is supported by the Natural Science Foundation of China (61703180) and by the Shandong Provincial Natural Science Foundation (ZR2016AM17, ZR2017MA043).

\section{Availability of data and materials}

The authors declare that all data and materials in the article are available and veritable.

\section{Competing interests}

The authors declare that they have no competing interests.

\section{Authors' contributions}

The authors declare that the study was realized in collaboration with the same responsibility. All authors read and approved the final manuscript.

\section{Publisher's Note}

Springer Nature remains neutral with regard to jurisdictional claims in published maps and institutional affiliations.

Received: 20 November 2018 Accepted: 21 February 2019 Published online: 28 February 2019

\section{References}

1. Jackson, F.: On q-functions and a certain difference operator. Trans. R. Soc. Edinb. 46, $253-281$ (1908)

2. Jackson, F.: On q-definite integrals. Pure Appl. Math. Q. 41, 193-203 (1910)

3. Kac, V., Cheung, P.: Quantum Calculus. Springer, New York (2002)

4. Agarwal, R.: Certain fractional $q$-integrals and q-derivatives. Proc. Camb. Philos. Soc. 66, 365-370 (1969)

5. Miao, F., Liang, S.: Uniqueness of positive solutions for fractional $q$-difference boundary value problems with p-Laplacian operator. Electron. J. Differ. Equ. 2013, 174, 1-11 (2013)

6. Graef, J., Kong, L.: Positive solutions for a class of higher order boundary value problems with fractional $q$-derivatives. Appl. Math. Comput. 218, 9682-9689 (2012)

7. Ahmad, B., Nietoa, J., Alsaedi, A., Al-Hutami, H.: Existence of solutions for nonlinear fractional q-difference integral equations with two fractional orders and nonlocal four-point boundary conditions. J. Franklin Inst. 351, 2890-2909 (2014)

8. Han, Z.L., Pan, Y.Y., Yang, D.W.: The existence and nonexistence of positive solutions to a discrete fractional boundary value problem with a parameter. Appl. Math. Lett. 36, 1-6 (2014)

9. Li, X.H., Han, Z.L., Li, X.C.: Boundary value problems of fractional q-difference Schrodinger equations. Appl. Math. Lett. 46, 100-105 (2015)

10. Chidouh, A., Torres, D.: Existence of positive solutions to a discrete fractional boundary value problem and corresponding Lyapunov-type inequalities. Opusc. Math. 38(1), 31-40 (2018)

11. Zhao, X., Ge, W.: Unbounded solutions for a fractional boundary value problems on infinite interval. Acta Appl. Math. $109,495-505(2010)$

12. He, L., Dong, X., Bai, Z., Chen, B.: Solvability of some two-point fractional boundary value problems under barrier strip conditions. J. Funct. Spaces 2017, Article ID 1465623 (2017)

13. Liang, S., Zhang, J.: Existence of three positive solutions of $m$-point boundary value problems for some nonlinear fractional differential equations on an infinite interval. Comput. Math. Appl. 61, 3343-3354 (2011)

14. Zhao, X., Zhao, D., Ge, W.: Solvability for fractional multi-point boundary value problems on half line. Ann. Differ. Equ. 27(3), 395-400 (2011)

15. Liu, X., Jia, M.: Multiple solutions for fractional differential equations with nonlinear boundary conditions. Comput. Math. Appl. 59, 2880-2886 (2010)

16. Cui, Y., Ma, W., Sun, Q., Su, X.: New uniqueness results for boundary value problem of fractional differential equation. Nonlinear Anal., Model. Control 23, 31-39 (2018)

17. Cui, Y., Sun, Q., Su, X.: Monotone iterative technique for nonlinear boundary value problems of fractional order $p \in(2,3]$. Adv. Differ. Equ. 2017, 248, 1-15 (2017)

18. Zhang, X., Zhong, Q.: Uniqueness of solution for higher-order fractional differential equations with conjugate type integral conditions. Fract. Calc. Appl. Anal. 20(6), 1471-1484 (2017)

19. Zhang, X., Zhong, Q.: Triple positive solutions for nonlocal fractional differential equations with singularities both on time and space variables. Appl. Math. Lett. 80, 12-19 (2018) 
20. $\mathrm{Xu}, \mathrm{M} ., \mathrm{Han}, \mathrm{Z}$.: Positive solutions for integral boundary value problem of two-term fractional differential equations. Bound. Value Probl. 2018, 100, 1-13 (2018)

21. Song, Q., Bai, Z:: Positive solutions of fractional differential equations involving the Riemann-Stieltjes integral boundary condition. Adv. Differ. Equ. 2018, 183 (2018)

22. Gamze Düzgün, F., lannizzotto, A.: Three nontrivial solutions for nonlinear fractional Laplacian equations. Adv Nonlinear Anal. 7(2), 211-226 (2018)

23. Giacomoni, J., Mukherjee, T., Sreenadh, K.: Positive solutions of fractional elliptic equation with critical and singular nonlinearity. Adv. Nonlinear Anal. 6(3), 327-354 (2017)

24. Lyons, J., Neugebauer, J.: Positive solutions of a singular fractional boundary value problem with a fractional boundary condition. Opusc. Math. 37(3), 421-434 (2017)

25. Annaby, M., Mansour, Z:: q-Fractional Calculus and Equations. Springer, New York (2012)

26. Rajković, P., Marinković, S., Stanković, M.: Fractional integrals and derivatives in q-calculus. Appl. Anal. Discrete Math $1(1), 311-323(2007)$

27. Li, X., Han, Z., Sun, S.: Existence of positive solutions of nonlinear fractional $q$-difference equation with parameter. Adv. Differ. Equ. 2013, 260 (2013)

28. Granans, A., Guenther, P., Lee, J: Some general existence principle in Carathéodory theory of nonlinear systems. J. Math. Pures Appl. 70, 153-196 (1991)

29. Su, X., Zhang, S.: Unbounded solutions to a boundary value problem of fractional order on the half-line. Comput. Math. Appl. 61, 1079-1087 (2011)

\section{Submit your manuscript to a SpringerOpen ${ }^{0}$ journal and benefit from:}

- Convenient online submission

- Rigorous peer review

Open access: articles freely available online

- High visibility within the field

Retaining the copyright to your article

Submit your next manuscript at $\boldsymbol{~ s p r i n g e r o p e n . c o m ~}$ 